\title{
Manipulating gene expression for the metabolic engineering of plants
}

\begin{abstract}
Introducing and expressing foreign genes in plants present many technical challenges that are not encountered with microbial systems. This review addresses the variety of issues that must be considered and the variety of options that are available, in terms of choosing transformation systems and designing recombinant transgenes to ensure appropriate expression in plant cells. Tissue specificity and proper developmental regulation, as well as proper subcellular localization of products, must be dealt with for successful metabolic engineering in plants.
\end{abstract}

Keyword: Gene expression; Metabolic engineering; Plants 